\title{
Weight Gain in Veterans Taking Duloxetine, Pregabalin, or Both for the Treatment of Neuropathy
}

\author{
Hannah Poppen, PharmD; Kelley Oehlke, PharmD, BCACP; and Emily Van Klompenburg, PharmD, BCACP
}

\begin{abstract}
Introduction: Peripheral neuropathy is a common condition with an estimated incidence of 3 million cases in the United States per year, with manifestations including weakness, numbness, burning or tingling sensations, and lingering pain. The burden of neuropathy may be greater among veterans due to the higher prevalence of type 2 diabetes mellitus (T2DM) and an aging population. Among the medications used to treat neuropathy are duloxetine and pregabalin. It has been observed at the Sioux Falls Veterans Affairs Health Care System (SFVAHCS) that veterans who are treated for neuropathy with duloxetine, pregabalin, or both, may experience significant weight gain after starting therapy. The purpose of this study was to evaluate the association of weight gain in veterans taking duloxetine, pregabalin, or both, for the treatment of neuropathy.
\end{abstract}

Methods: This was a retrospective, chart review study conducted at the SFVAHCS. The primary end point of this study was the change in body weight, expressed in pounds, after 12 to 18 months of treatment. The secondary end points of this study were the percent change in body weight; duration effect; dose effect, which evaluated weight gain at doses of duloxetine $>60 \mathrm{mg} / \mathrm{d}$ and pregabalin at doses > $300 \mathrm{mg} / \mathrm{d}$; change in hemoglobin $A_{1 c}$ in patients with prediabetes and T2DM, and involvement in the
Managing Overweight Veterans Everywhere (MOVE!) weight management program.

Results: The change in body weight after 12 to 18 months of treatment was $-0.8 \mathrm{lb}$ in the duloxetine group, $+2.9 \mathrm{lb}$ in the pregabalin group, and $+5.5 \mathrm{lb}$ in the pregabalin plus duloxetine group $(P=.12)$. The change in body weight after $>12$ months of treatment was $-0.88 \mathrm{lb}$ in the duloxetine group, $+3.6 \mathrm{lb}$ in the pregabalin group, and $+8.5 \mathrm{lb}$ in the duloxetine plus pregabalin group $(P=.046)$. The change in body weight in patients who received increased doses of the study agents was $-2.8 \mathrm{lb}$ in the duloxetine group and $+6.5 \mathrm{lb}$ in the pregabalin group $(P=.047)$.

Conclusions: There was no significant difference in weight in veterans who took duloxetine, pregabalin, or both, for treatment of neuropathy after 12 to 18 months of therapy. However, there was a difference in weight gain among the 3 groups when therapy lasted $>12$ months. The combination therapy of pregabalin and duloxetine was associated with the most amount of weight gain, followed by pregabalin alone. Monotherapy of duloxetine had minimal association with weight gain. In veterans who took increased doses of duloxetine or pregabalin, there was a difference in weight between the monotherapy groups, with pregabalin associated with weight gain and duloxetine associated with weight loss.
Author affiliations can be found at the end of the article.

Correspondence:

Hannah Poppen

(h.poppen7@gmail.com)

Fed Pract. 2021;38(5). Published online May 12. doi:10.12788/fp.0111
$\mathrm{N}$ europathy is the result of damage to the nervous system. This dysfunction generally occurs in peripheral nerves, which are the circuits that transmit signals to the brain and spinal cord. The peripheral nervous system is responsible for controlling motor and autonomic nerves and conduction of sensory information. Injury to the nervous system can lead to changes in nerve fiber sensitivity and malfunctioning of nerve stimuli pathways. Neuropathy may be a sequela of a wide variety of diseases, including diabetes mellitus (DM), autoimmune disorders, infections, and cancer. Also, neuropathy can be caused by medications, trauma, exposure to toxins, classified idiopathic. ${ }^{1-5}$

Peripheral neuropathy is a common condition with an estimated incidence of $>3$ million cases in the United States per year. ${ }^{4}$ The burden of neuropathy may be greater among veterans, due to a higher prevalence of type $2 \mathrm{DM}$ (T2DM) and an aging population. Manifestations of neuropathy include weakness, numbness, burning or tingling sensations, and lingering pain. ${ }^{3,5}$ This can lead to limited mobility and decreased quality of life. Neuropathy can be debilitating, but several medications can be used to alleviate symptoms-including duloxetine and pregabalin. The American Diabetes Association recommends either agent as initial treatment for neuropathic pain in patients with DM. ${ }^{2}$ As with all medication use, the benefits and risks of treatment must be assessed prior to initiation of therapy.

The Centers for Disease Control and Prevention estimates $>70 \%$ of adults in the United States are overweight or obese. ${ }^{6}$ Excessive weight gain causes a higher risk of developing certain comorbidities, such as coronary artery disease, cerebrovascular accident, T2DM, and cancer, and all can lead to premature death. It is important to avoid excessive weight gain whenever 
possible, especially in patients already at a high risk for developing these diseases.

The correlation of weight gain in patients taking duloxetine, pregabalin, or both is not well studied. Duloxetine has the potential to cause weight gain or weight loss, with reports of $>1 \%$ incidence for either effect. ${ }^{7}$ Clinical significance of weight changes caused by duloxetine is uncertain. Pregabalin is more likely to cause weight gain, with a reported incidence between 2 and $14 \% .{ }^{8}$ Weight gain may be associated with dose and duration; 1 study demonstrated an average weight gain of about $11 \mathrm{lb}$ after 2 years of pregabalin treatment. ${ }^{8}$ The medical literature lacks information regarding weight gain associated with combination therapy of duloxetine and pregabalin. The objective of this study was to investigate the association of weight gain in veterans taking duloxetine, pregabalin, or both for the treatment of neuropathy.

\section{METHODS}

A retrospective, single-center, chart review was conducted at the Sioux Falls Veterans Affairs Health Care System (SFVAHCS). Data were collected through manual chart review of US Department of Veterans Affairs (VA) electronic health records (EHRs). Patients included were veterans aged 18 to 89 years who were initiated on duloxetine and/or pregabalin between October 2015 and September 2018. The monotherapy groups were made equal by randomization based on whichever group had the lesser number of participants. The indication of neuropathy was determined by the presence of neuropathic pain, neuropathy, nerve pain, or otherwise similar terminology in the prescription directions for use in the EHR. Patients were excluded if they did not have an active prescription of the study agent(s) for $\geq 12$ months or if there was a lack of documented body weight(s) at baseline and/or at followup outpatient visit(s) occurring 12 to 18 months after initiation of therapy. Additional exclusion criteria were health conditions, including active cancer; pregnancy; history of bariatric surgery; if the patient received hospice care; was morbidly obese (body mass index [BMI] > 40; or if estimated glomerular filtration rate (eGFR)
FIGURE Change in Body Weight After 12 to 18 Months of Treatment ${ }^{\mathrm{a}}$

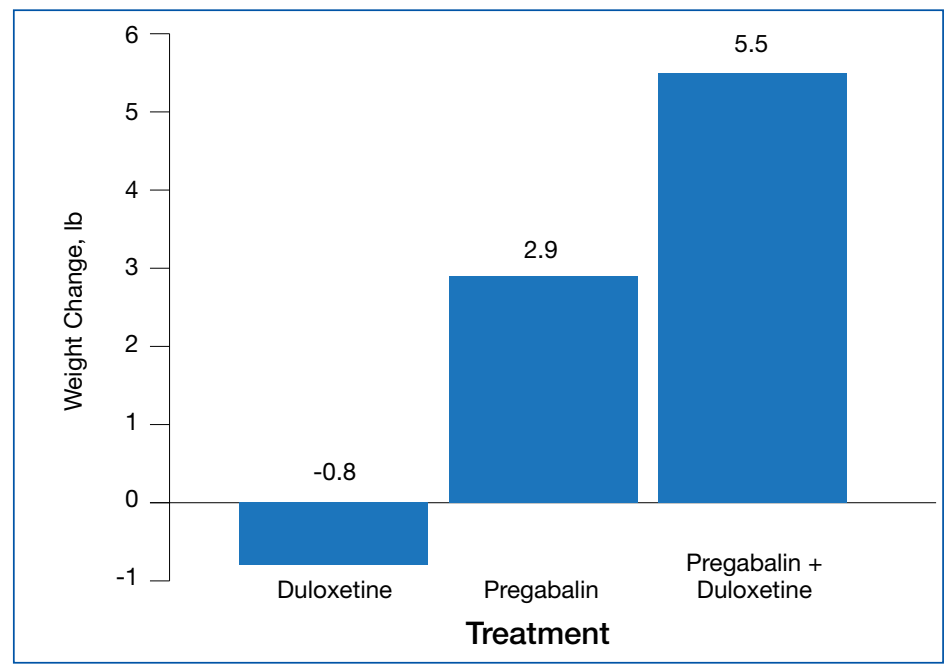

${ }^{\mathrm{a} P}=.12$.

was $<30 \mathrm{~mL} / \mathrm{min} / 1.83 \mathrm{~m}^{2}$. Patients also were excluded if they were concurrently taking any of the following medications: antipsychotics, tricyclic antidepressants, venlafaxine, divalproex/valproic acid, lithium, mirtazapine, weight loss medications (orlistat, lorcaserin, phenterminetopiramate, naltrexone-bupropion, liraglutide), chronic corticosteroids, or chronic opioids; chronic being defined as receiving more than a 30-day supply.

The primary end point of this study was the change in body weight, expressed in pounds, after 12 to 18 months of treatment. If multiple weights were obtained during the 12- to 18 -month period, the weight recorded closest to 12 months was used. The secondary end points included the percent change in body weight and dose effect, which evaluated change in weight at doses of duloxetine $>60 \mathrm{mg} / \mathrm{d}$, and pregabalin at doses $>300 \mathrm{mg} / \mathrm{d}$. Duration of effect was evaluated as a secondary end point; contrary to the primary end point, the weight furthest from 12 months was recorded. The change in hemoglobin $\mathrm{A}_{1 \mathrm{c}}\left(\mathrm{HbA}_{1 \mathrm{c}}\right)$ in patients with prediabetes and $\mathrm{DM}$ also was investigated as a secondary end point. Last, involvement in the Managing Overweight Veterans Everywhere (MOVE!) weight management program at SFVAHCS and its effect on weight gain was reviewed. 
TABLE 1 Baseline Characterisics

\begin{tabular}{lcccc} 
& $\begin{array}{c}\text { Duloxetine } \\
(\mathbf{n}=\mathbf{7 7})\end{array}$ & $\begin{array}{c}\text { Pregabalin } \\
(\mathbf{n}=\mathbf{7 7})\end{array}$ & $\begin{array}{c}\text { Pregabalin }+ \\
\text { Duloxetine }(\mathbf{n}=\mathbf{2 0})\end{array}$ & $\begin{array}{c}\boldsymbol{P} \\
\text { value }\end{array}$ \\
\hline Age, mean, y & 67 & 65 & 63 & .32 \\
\hline Male, No. (\%) & $68(88.3)$ & $72(92.3)$ & $18(90)$ & .54 \\
\hline Race, White, No. (\%) & $61(79.2)$ & $69(89.6)$ & $19(95)$ & .08 \\
\hline Weight, mean, lb & 213.1 & 211.5 & 224.4 & .35 \\
\hline Body mass index, mean & 30.8 & 30.7 & 32.2 & .46 \\
\hline eGFR, mean, mL/min/1.83m² & 77.9 & 82 & 83.2 & .38 \\
\hline Diabetes mellitus, No. (\%) & $32(41.6)$ & $43(55.8)$ & $10(50)$ & .23
\end{tabular}

Abbreviation: eGFR, estimated glomerular filtration rate.

Baseline characteristics were collected to determine the variability between each study group. Data collected during the study included age, sex, race, weight, $\mathrm{BMI}, \mathrm{HbA}_{1 \mathrm{c}}$, eGFR, DM diagnosis, insulin therapy prescription, duration of use, and MOVE! program participation.

\section{Statistical Analysis}

The primary and secondary end points were analyzed using an analysis of variance statistical test. Results were considered statistically significant at $P<.05$.

\section{RESULTS}

A total of 174 participants were included in this study, with 77 in each monotherapy group, and 22 in the combination therapy group. More than 300 patients were excluded from the study due to prespecified inclusion and exclusion criteria. Baseline characteristics were similar among the 3 groups, with no statistically significant differences identified (Table 1).

\section{Primary End Point}

The change in body weight after 12 to 18 months of treatment was $-0.8 \mathrm{lb}$ in the duloxetine group, $+2.9 \mathrm{lb}$ in the pregabalin group, and $+5.5 \mathrm{lb}$ in the pregabalin plus $\mathrm{du}-$ loxetine group $(P=.12)$ (Figure).

\section{Secondary End Points}

The percent change in body weight after 12 to 18 months of treatment was $-0.3 \%$ in the duloxetine group, $+1.5 \%$ in the pregabalin group, and $+2.0 \%$ in the dulox- etine plus pregabalin group $(P=.18)$. The change in body weight beyond 12 months of treatment was $-0.9 \mathrm{lb}$ in the duloxetine group, $+3.6 \mathrm{lb}$ in the pregabalin group, and $+8.5 \mathrm{lb}$ in the duloxetine plus pregabalin group $(P=.05)$. The change in $\mathrm{HbA}_{1 \mathrm{c}}$ in patients with DM and pre-DM was $-0.1 \%$ in the duloxetine group, $+0.3 \%$ in the pregabalin group, and $-0.3 \%$ in the duloxetine plus pregabalin group $(P=.14)$. The change in body weight in patients who received increased doses of the study agents was $-2.8 \mathrm{lb}$ in the duloxetine group and $+6.5 \mathrm{lb}$ in the pregabalin group $(P=.05)$. Among veterans who participated in MOVE!, change in body weight after 12 to 18 months of treatment was $+1.5 \mathrm{lb}$ in the duloxetine group, $+4.9 \mathrm{lb}$ in the pregabalin group, and $+3.4 \mathrm{lb}$ in the pregabalin plus duloxetine group $(P=.91)$ (Table 2$)$.

\section{DISCUSSION}

The purpose of this retrospective chart review was to evaluate the association of weight gain in veterans taking duloxetine and/or pregabalin for the treatment of neuropathy. Although the primary end point, weight gain after 12 to 18 months of therapy, was not statistically significant, we found notable trends and associations worthy of discussion.

The secondary end point of the difference in weight gain in veterans taking duloxetine, pregabalin, or both for a treatment duration $>12$ months was statistically significant. For this secondary end point, the weight recorded was when the study agent(s) were discontinued or the most recent weight obtained if participants still had an active prescription; the average duration of treatment in the 3 study groups was about 24 months. These weights differed from the primary end point, in which weight closest to 12 months of therapy was recorded.

The other secondary end point that was statistically significant was the difference in weight gain in patients who were on higher doses of duloxetine or pregabalin. This specifically examined participants who were on doses of duloxetine $>60 \mathrm{mg} / \mathrm{d}$ and pregabalin $>300 \mathrm{mg} / \mathrm{d}$. Duloxetine was associated with weight loss, whereas pregabalin was associated with weight gain, with a difference 
TABLE 2 Secondary End Points

\begin{tabular}{lcccc} 
Criteria & Duloxetine & Pregabalin & $\begin{array}{c}\text { Pregabalin }+ \\
\text { Duloxetine }\end{array}$ & $\begin{array}{c}\boldsymbol{P} \\
\text { value }\end{array}$ \\
\hline $\begin{array}{l}\text { Weight change, \% } \\
\text { Weight change after at least 12 months, lb }\end{array}$ & $\begin{array}{c}(\mathrm{n}=77) \\
-0.3\end{array}$ & $\begin{array}{c}(\mathrm{n}=77) \\
1.5 \\
+3.6\end{array}$ & $\begin{array}{c}(\mathrm{n}=20) \\
+2.0 \\
+8.5\end{array}$ & .18 \\
\hline $\begin{array}{l}\text { Hemoglobin } \mathrm{A}_{1 \mathrm{C}} \text { change in and patients with } \\
\text { prediabetes or diabetes mellitus, \% }\end{array}$ & $\begin{array}{c}(\mathrm{n}=43) \\
-0.1\end{array}$ & $\begin{array}{c}(\mathrm{n}=50) \\
+0.3\end{array}$ & $\begin{array}{c}(\mathrm{n}=12) \\
-0.3\end{array}$ & .14 \\
\hline $\begin{array}{l}\text { Weight change for patients prescribed } \\
\text { duloxetine }>\text { 60 mg/d or pregabalin > 300 mg/d, lb }\end{array}$ & $\begin{array}{c}(\mathrm{n}=13) \\
-2.8\end{array}$ & $\begin{array}{c}(\mathrm{n}=21) \\
+6.5\end{array}$ & --- \\
\hline $\begin{array}{l}\text { Weight change for patients in Managing Overweight } \\
\text { Veterans Everywhere program, lb }\end{array}$ & $\begin{array}{c}(\mathrm{n}=10) \\
+1.5\end{array}$ & $\begin{array}{c}(\mathrm{n}=8) \\
+4.9\end{array}$ & $\begin{array}{c}(\mathrm{n}=5) \\
+3.4\end{array}$ & .05 \\
\hline
\end{tabular}

of about $10 \mathrm{lb}$ between the groups. The significance of this secondary end point demonstrates that increased doses of duloxetine and pregabalin are more associated with changes in weight compared with standard doses.

The secondary end points of percent change in body weight, change in HBA in patients with DM and prediabetes, and weight gain in patients who participated in the MOVE! weight management program were not statistically significant among the 3 study groups. Given the relatively small sample sizes, more significant differences in the evaluation of the primary and secondary end points may have been observed with a larger patient population.

Study investigators made additional observations beyond the primary and secondary end points. Most notably, $>300$ patients were excluded from this study because they did not continue treatment beyond 12 months. The investigators found this number staggering, as it may imply that veterans were not satisfied with treatment agent(s) within 1 year of initiation, which could be due to lack of efficacy or intolerable adverse effects.

The mechanism of why combination therapy of duloxetine and pregabalin may be more associated with weight gain compared with either agent alone is unknown. Since this study found duloxetine to be more associated with weight loss, the mechanism does not seem to be an additive effect. The alternative hypothesis proposed prior to the completion of this study stemmed from an observation seen by health care providers at SFVAHCS.

\section{Limitations}

The retrospective nature of the study does not provide proof of causation but does demonstrate association. There was no control group, and the study design did not allow for randomization of participants. Additionally, since the study was completed at a single center, there was potential for selection bias. Future studies could benefit from pursuing a multicenter study design, which may provide a higher level of external validity. There are several confounding factors that have the potential to influence changes in weight, all of which cannot feasibly be accounted for. Since participants were ambulatory veterans, medication adherence could not be confirmed.

\section{CONCLUSIONS}

There was no difference in weight gain in veterans who took duloxetine, pregabalin, or both for treatment of neuropathy after 12 to 18 months of therapy. However, there was a difference in weight gain between the 3 groups when therapy lasted $>12$ months. The combination therapy of pregabalin and duloxetine was associated with the most amount of weight gain, followed by pregabalin alone. Duloxetine monotherapy had minimal impact on weight.

In veterans who took increased doses of duloxetine or pregabalin, there was a statistically significant difference in weight between the monotherapy groups, with pregabalin associated with weight gain and duloxetine associated with weight loss.

For patients in which weight gain may be a concern, it would be reasonable to prefer duloxetine rather than pregabalin for 
initial treatment of neuropathy. Pregabalin should be used at the lowest effective dose to minimize risk of weight gain. Combination therapy of duloxetine and pregabalin for the treatment of neuropathy seems to be associated with the most amount of weight gain compared with either therapy alone. Association of changes in weight is greater as treatment duration lasts beyond 12 months.

\section{Author affiliations}

Hannah Poppen was a PGY1 Pharmacy Resident at the time the article was written; Kelley Oehlke is Associate Chief of Pharmacy, both at Sioux Falls VA Health Care System in South Dakota. Emily Van Klompenburg is an Assistant Professor at South Dakota State University College of Pharmacy and Allied Health Professions in Sioux Falls.

\section{Author disclosures}

The authors report no actual or potential conflicts of interest with regard to this article.

\section{Disclaimer}

The opinions expressed herein are those of the authors and do not necessarily reflect those of Federal Practitioner, Frontline Medical Communications Inc., the US Government, or any of its agencies. This article may discuss unlabeled or investigational use of certain drugs. Please review the complete prescribing information for specific drugs or drug combina- tions -including indications, contraindications, warnings, and adverse effects-before administering pharmacologic therapy to patients.

\section{References}

1. Onakpoya IJ, Thomas ET, Lee JJ, Goldacre B, Heneghan CJ. Benefits and harms of pregabalin in the management of neuropathic pain: a rapid review and meta-analysis of randomised clinical trials. BMJ Open. 2019;9(1):e023600. Published 2019 Jan 21. doi:10.1136/bmjopen-2018-023600

2. American Diabetes Association. 11. Microvascular Complications and Foot Care: Standards of Medical Care in Diabetes-2019. Diabetes Care. 2019;42(suppl 1):S124-S138. doi:10.2337/dc19-S011

3. Baumann TJ, Herndon CM, Strickland JM. Pain Management. In: DiPiro JT, Talbert RL, Yee GC, Matzke GR, Wells BG, Posey LM, eds. Pharmacotherapy: A Pathophysiologic Approach. 9th ed. New York, NY: McGraw-Hill; 2014:925.

4. National Institute of Neurological Disorders and Stroke. Peripheral neuropathy fact sheet. Updated March 16 , 2020. Accessed March 10, 2021. https://www.ninds.nih .gov/Disorders/Patient-Caregiver-Education/Fact-Sheets /Peripheral-Neuropathy-Fact-Sheet

5. Feldman EL. Patient education: diabetic neuropathy (beyond the basics). Updated January 20, 2021. Accessed April 21, 2021. https://www.uptodate.com/contents /diabetic-neuropathy-beyond-the-basics

6. Centers for Disease Control and Prevention. Overweight and obesity. Updated October 29, 2020. Accessed March 10, 2021. https://www.cdc.gov/obesity/index.html

7. Cymbalta (duloxetine) [prescribing information]. Eli Lilly and Company; April 2020.

8. Lyrica (pregabalin) [prescribing information]. Parke-Davis, Division of Pfizer Inc; June 2020. 